\title{
FORTBILDUNG
}

\author{
Schwerpunkt \\ Hypertonie
}

27_ Kombinieren wir zu spät?

30_ Lebensqualität unter Hochdrucktherapie

34_Wie viel Sport ist genug?

"Hypertension 2008"

\section{Berlin unter Hochdruckeinfluss}

\section{Prof. Dr. med. Thomas Unger}

Charité - Universitätsmedizin Berlin

Chair, Scientific Programme Committee

Hypertension 2008 Berlin

— Vom 14. bis 19. Juni 2008 findet in Berlin der internationale Hypertoniekongress „Hypertension 2008“ statt, der von drei Hypertoniegesellschaften gemeinsam veranstaltet wird: der European Society of Hypertension (ESH), der International Society of Hypertension (ISH) und der Deutschen Hypertonie-Liga (DHL). Mit seinem umfangreichen wissenschaftlichen Hauptprogramm, den vielen Satellitensymposien und den zahlreichen Fortbildungseinheiten gibt dieser Kongress sowohl einen Überblick über den neuesten Erkenntnisstand der theoretischen und klinischen Bluthochdruckforschung als auch über gegenwärtige Konzepte in Diagnostik und Therapie der Hypertonie sowie verwandter kardiovaskulärer und metabolischer Erkrankungen.

In den neun Hauptvorträgen kommen so unterschiedliche Themen zur Sprache wie die Rolle des neu entdeckten Reninrezeptors, die Pathophysiologie vaskulärer Erkrankungen, molekularbiologische Grundlagenerkenntnisse vaskulärer Krankheiten wie z.B. die Bedeutung der MicroRNA oder der nukleären Transkriptionsfaktoren, aber auch aktuelle klinisch-therapeutische Fragen wie die Stammzelltherapie kardiovaskulärer Erkrankungen, der Stellenwert und die Erarbeitungskriterien von therapeutischen Empfehlungen und Leitlinien und die Zukunft von Evidenz generierenden klinischen Studien. Die über dreißig parallel stattfindenden freien Vortragssitzungen werden jeweils von einer eingeladenen „State-of-the-Art“-Vorlesung gekrönt. Die Themen reichen von der Albuminurie über die vaskuläre Plastizität, das metabolische Syndrom, geschlechtsspezifische Aspekte der Hypertonie, klinische Alltagsfragen in Diagnostik und Therapie bis zur Epidemiologie von Hypertonie und metabolisch-vaskulären Erkrankungen in der Dritten Welt. Eine Reihe dieser Sitzungen befasst sich darüber hinaus mit den neuesten Ergebnissen großer klinischer Studien, die in diesem Jahr veröffentlicht wurden und werden wie ONTARGET, ADVANCE, HYVET, PRoFESS u.a.

Auf einer von der DHL veranstalteten Sondersitzung am Donnerstagmittag (19. Juni) werden die wissenschaftlichen Höhepunkte des Kongresses in deutscher Sprache vorgestellt. Wer sich nur für diese Sitzung interessiert, kann mit einer Tageskarte am Kongress teilnehmen.

Für die Ärzte aus Berlin und Umgebung wird am Samstag, den 14. Juni um 9.30 Uhr zusammen mit der Berliner Ärztekammer ein Symposium in deutscher Sprache stattfinden, in dem praktisch-relevante Fragen rund um die Hypertonie erörtert werden. Für die Berliner Bevölkerung veranstaltet die DHL am gleichen Vormittag in der „Urania“ einen Hypertonie-Gesundheitstag.

\section{Noch immer viel zu viele Hypertoniker}

Ist ein derartiger Aufwand eigentlich gerechtfertigt? Ich bin der Überzeugung: ja, vielleicht mehr denn je. Wie wir alle wissen, haben wir das klinische Problem der Hypertonie immer noch nicht richtig im Griff, weder in der Forschung noch in der Therapie, insbesondere was die Kontrolle des hohen Blutdrucks in der Bevölkerung angeht. Noch immer erkranken viel zu viele Menschen, zunehmend in den industriellen Schwellenländern, aber auch bei uns in Europa an den Folgen der Hypertonie. Noch immer gibt es viel zu viele Schlaganfälle, die wesentlich durch den hohen Blutdruck verursacht sind, von den anderen Hypertoniefolgen an Gefäßen, Niere und Herz ganz zu schweigen.

Der wissenschaftliche Austausch, aber auch die fortbildende Weitergabe des erarbeiteten und abgesicherten Wissens, wie sie ein derartiger Kongress auf höchstem internationalem Niveau anbietet, sind notwendige Voraussetzungen, um unserem gemeinsamem Ziel näher zu kommen, nämlich die kardiovaskulär-renalen und metabolischen Störungen und Erkrankungen möglichst zu verhüten oder zumindest rechtzeitig zu erkennen und zu behandeln.

Ich hoffe, Ihr Interesse an „Hypertonie 2008“ geweckt zu haben. Diesem Zweck dienen natürlich auch die nachfolgenden Artikel meiner Kollegen über drei verschiedene aktuelle Themen aus dem Hypertoniebereich.

Eine detaillierte Programmübersicht zum Kongress finden Sie im Internet unter www.hypertension2008.com. 